\title{
In silico screening of carbon-capture materials
}

Li-Chiang Lin ${ }^{1,2 \uparrow}$, Adam H. Berger ${ }^{3 \pi}$, Richard L. Martin ${ }^{4 \uparrow}$, Jihan Kim ${ }^{2 \uparrow}$, Joseph A.

Swisher $^{1,2}$, Kuldeep Jariwala ${ }^{2}$, Chris H. Rycroft ${ }^{4},{ }^{5}$, Abhoyjit S. Bhown $^{3}$, Michael W.

Deem ${ }^{6}$, Maciej Haranczyk ${ }^{4}$, and Berend Smit ${ }^{1,2,7}$

${ }^{1}$ Department of Chemical and Biomolecular Engineering, University of California, Berkeley, Berkeley, CA 94720-1462, USA

${ }^{2}$ Materials Science Division, Lawrence Berkeley National Laboratory, Berkeley, CA 94720, USA

${ }^{3}$ Electric Power Research Institute (EPRI), 3420 Hillview Avenue, Palo Alto, CA 94304, $U S A$

${ }^{4}$ Computational Research Division, Lawrence Berkeley National Laboratory, Berkeley, CA 94720-8139, USA

${ }^{5}$ Department of Mathematics, University of California, Berkeley, Berkeley, CA 94720 , $U S A$

${ }^{6}$ Departments of Bioengineering and Physics \& Astronomy, Rice University, Houston, TX 77005, USA

${ }^{7}$ Department of Chemistry, University of California, Berkeley, Berkeley, CA 94720-1462, $U S A$

"These authors contributed equally to this work 


\section{Uncertainties in The Energy Cost of Capturing Carbon}

To analyze the effect of these uncertainties on the overall parasitic energies, we selected a set of materials that spanned the range of parasitic energies. To simulate the propagation of possible errors on the thermodynamic input parameters in the parasitic energy, we changed each of these values by multiplying the actual value of a parameters by a factor, which was randomly selected from the interval $[0.8,1.2]$, i.e., a maximum possible error is plus or minus $20 \%$ on each of the thermodynamic variables. In this way, we generated, for each of the selected parasitic energies, 25 different sets of parameters. Figure SI 1 shows how these uncertainties propagate for a given value of the parasitic energy. We see that for high values of the parasitic energy, the results are much more sensitive. The reason is that small changes in the Henry coefficient have a large effect on the parasitic energy. In contrast, for low values of the parasitic energy the results are robust. This is consistent with the observation that we have for these materials a very broad optimum. Hence, some variations in the parameters have little influence, as at slightly different conditions a very similar optimal parasitic energy can be found. As we are mainly interested in materials with a low parasitic energy, this analysis shows that a $20 \%$ uncertainty in the main thermodynamic parameters should not have a significant influence on our estimates of the parasitic energy.

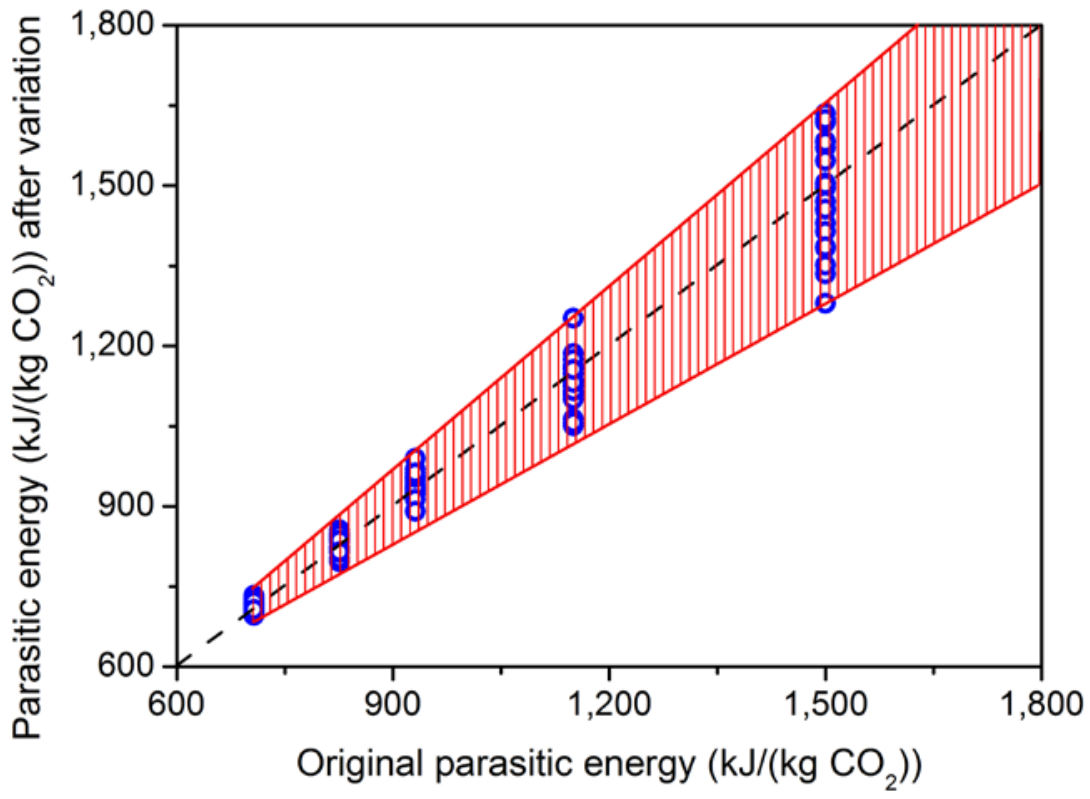

Figure SI 1: Uncertainties in the estimates of the parasitic energies. The blue dots are the 25 parameters sets for which we recalculated the parasitic energy after a change of $+/-20 \%$ of all parameters. The red line gives the upper and lower bounds of the errors in these sets. 


\section{Database of carbon capture materials}

We have collected all results obtained in this work in a database, accessible at www.carboncapturematerials.org. The investigated materials have been characterized in terms of:

- Their pore geometry (pore measured by the diameter of the largest included and free spheres, accessible volume and surface areas),

- Adsorption properties (Henry coefficients and heats of adsorption for $\mathrm{CO}_{2}$ and $\mathrm{N}_{2}$, estimated Langmuir isotherms and in some cases simulated isotherms),

- Performance using the model discussed in the article (including parasitic energy, working capacity, and $\mathrm{CO}_{2}$ purity).

The web interface allows the user to search for specific materials or materials with certain properties, and provides a graphical interface to browse the results. For example, plotting the parasitic energy as a function of the $\mathrm{CO}_{2}$ Henry coefficient gives Figure $2 \mathrm{a}$. In this figure each point is clickable and takes the user to the material entry page, which contains all the relevant properties for a particular material. 


\section{Detailed acknowledgements}

M.H., R.L.M. and C.H.R. were supported in part by the U.S. Department of Energy under contract DE-AC02-05CH11231. R.L.M and M.H. were supported jointly by DOE Office of Basic Energy Sciences and the Office of Advanced Scientific Computing Research through SciDAC project \#CSNEW918 entitled "Knowledge guided screening tools for identification of porous materials for $\mathrm{CO}_{2}$ separations".

B.S. was supported as part of the Center for Gas Separations Relevant to Clean Energy Technologies, an Energy Frontier Research Center funded by the U.S. Department of Energy, Office of Science, Office of Basic Energy Sciences under Award Number DESC0001015.

J.K. was supported by the Director, Office of Science, Advanced Scientific Computing Research, of the U.S. Department of Energy under Contract No. DE-AC02-05CH11231.

M.W.D. was supported by the U.S. Department of Energy under grant DE-FG0203ER15456.

J.S. and L-C.L. were supported by the Advanced Research Projects Agency - Energy (ARPA-E), U.S. Department of Energy.

K.J was supported in part by the U.S. Department of Energy under contract DE-AC0205CH1 1231 through the Carbon Capture Simulation Initiative (CCSI)

A.H.B. and A.S. B. were supported by Office of Innovation, Electric Power Research Institute.

This research used resources of the National Energy Research Scientific Computing Center, which is supported by the Office of Science of the U.S. Department of Energy under Contract No. DE-AC02-05CH11231. All of the GPU simulations were conducted on Dirac, which is a GPU cluster at NERSC that consists of 44 Fermi Tesla C2050 cards.

This work was prepared as an account of work sponsored by an agency of the United States Government. Neither the United States Government nor any agency thereof, nor any of their employees, makes any warranty, express or implied, or assumes any legal liability or responsibility for the accuracy, completeness, or usefulness of any information, apparatus, product, or process disclosed, or represents that its use would not infringe privately owned rights. Reference herein to any specific commercial product, process, or service by trade name, trademark, manufacturer, or otherwise does not necessarily constitute or imply its endorsement, recommendation, or favoring by the United States Government or any agency thereof. The views and opinions of authors expressed herein do not necessarily state or reflect those of the United States Government or any agency thereof. 


\section{Mixture Isotherms}

The figures below show a comparison of the mixture isotherms as computed by molecular simulations (dots) with those predicted by the competitive isotherm model (lines) for a set of representative zeolite structures. The blue lines and symbols give the isotherms for $\mathrm{N}_{2}$ and red lines and symbols the isotherms for $\mathrm{CO}_{2}$.

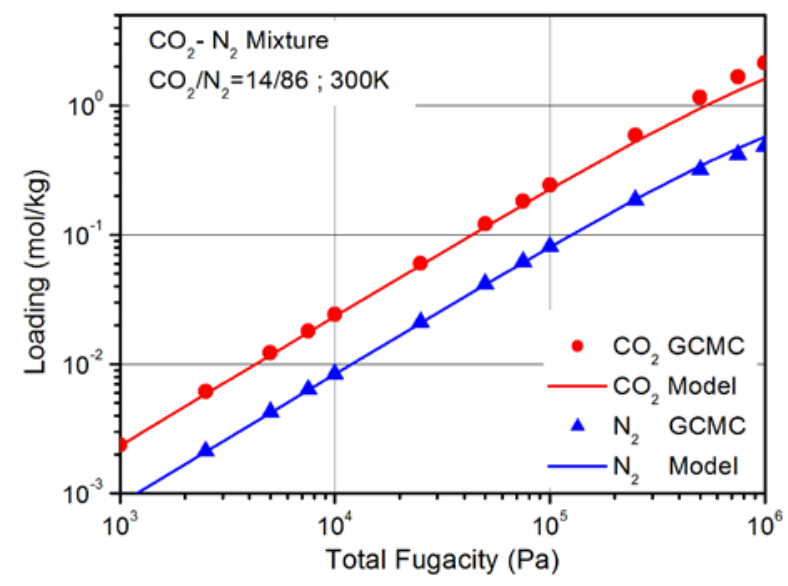

AEI

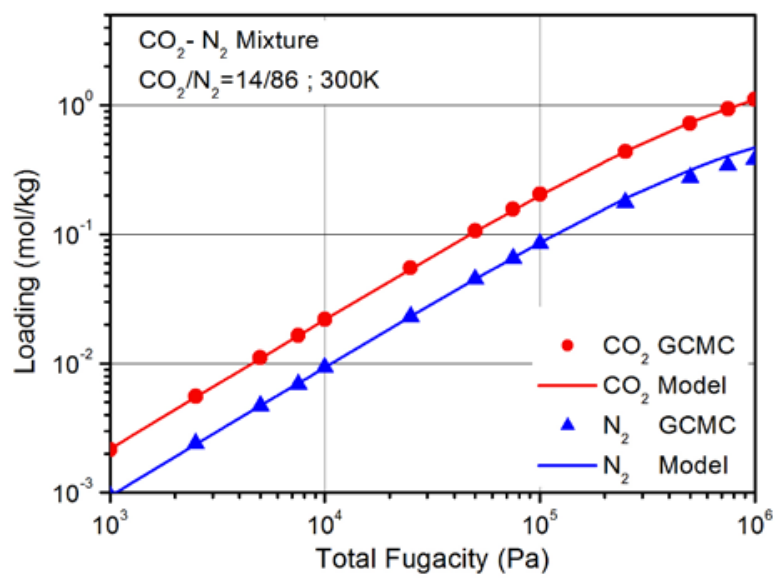

ZON 


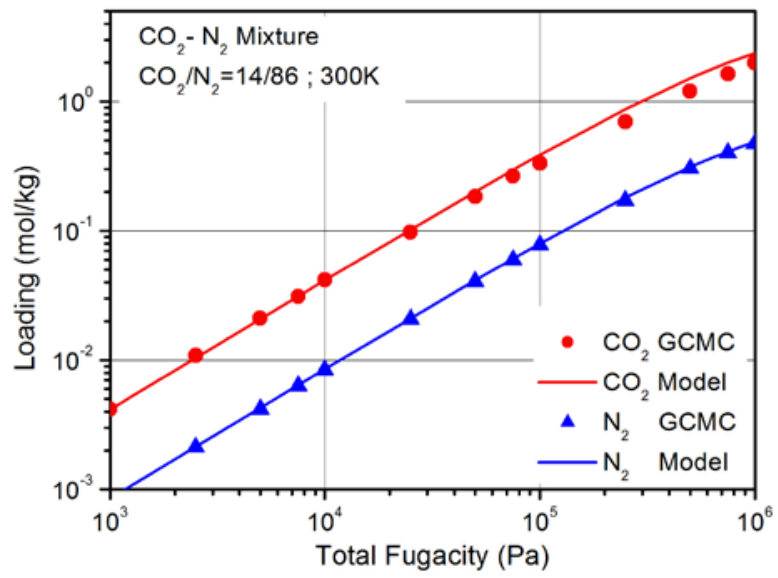

AFT

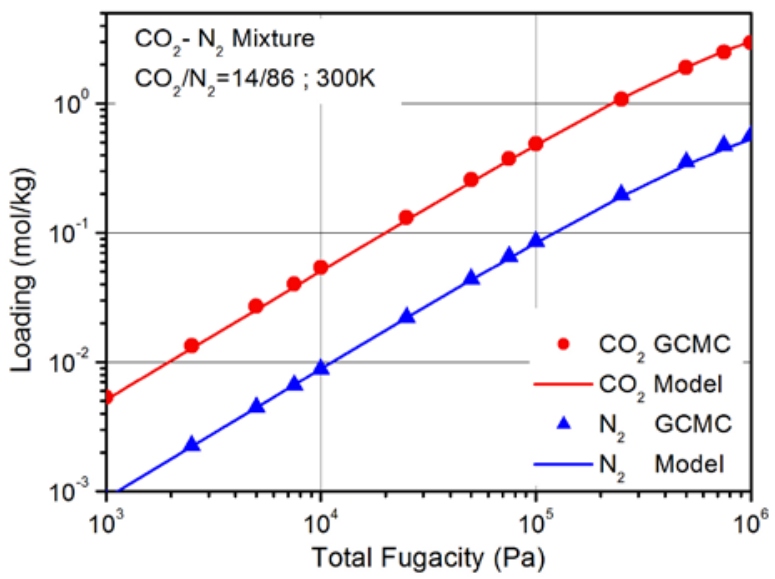

AFY 


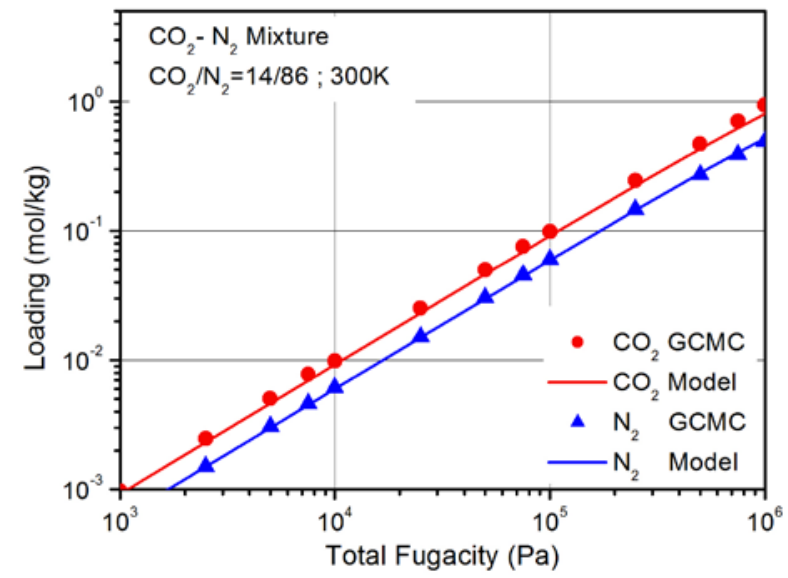

LTA

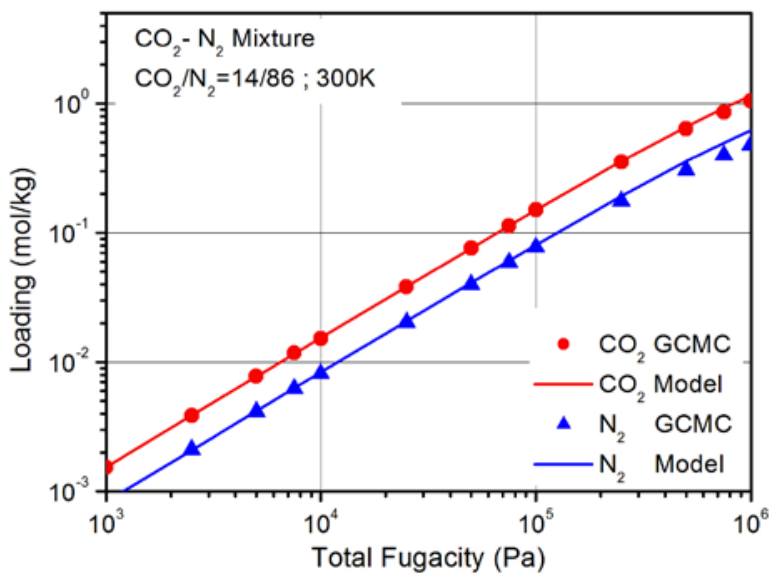

MWW 


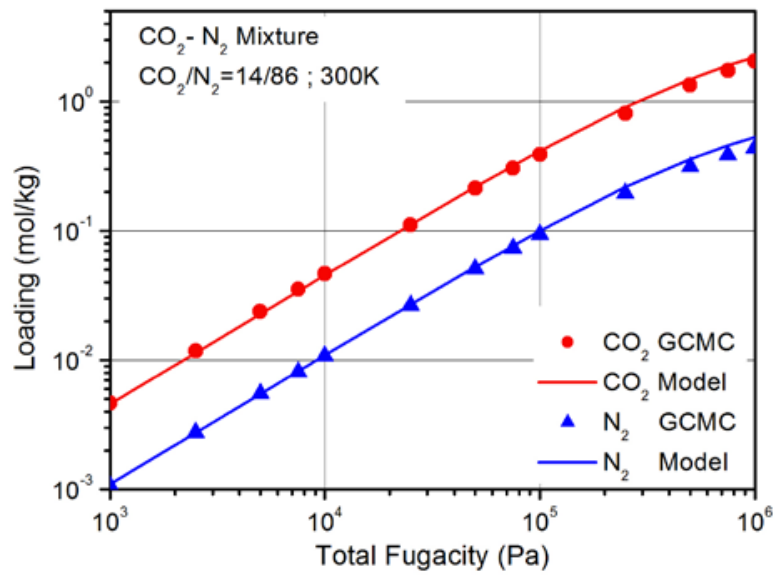

EAB

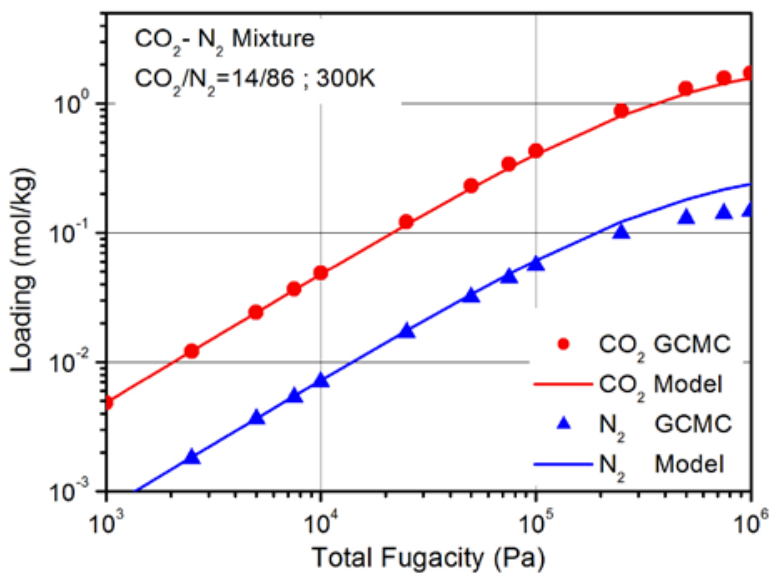

ASV 


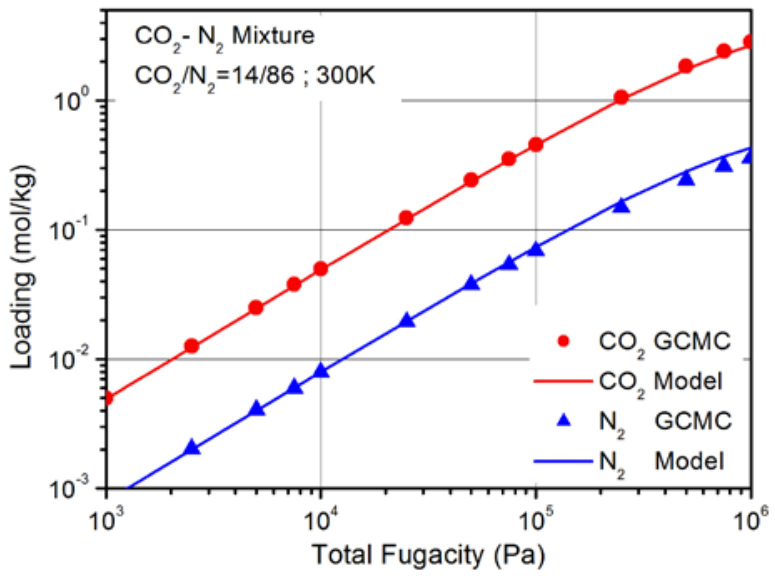

KFI

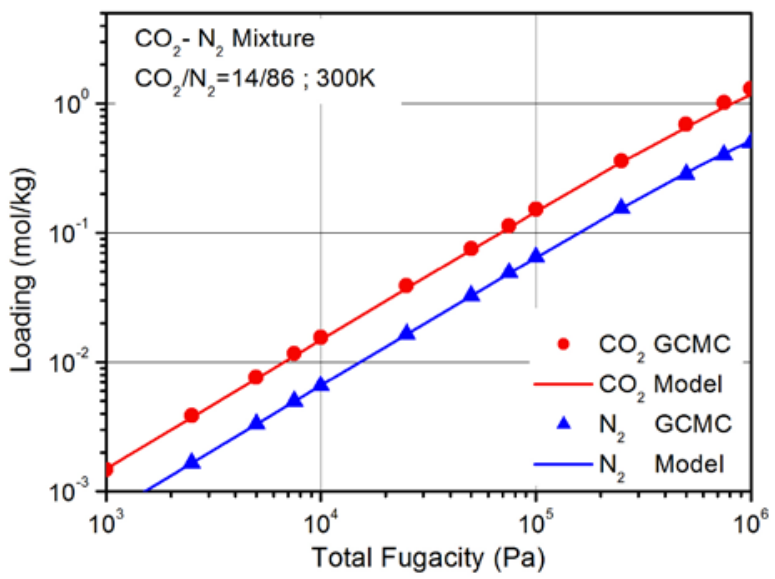

AFR 


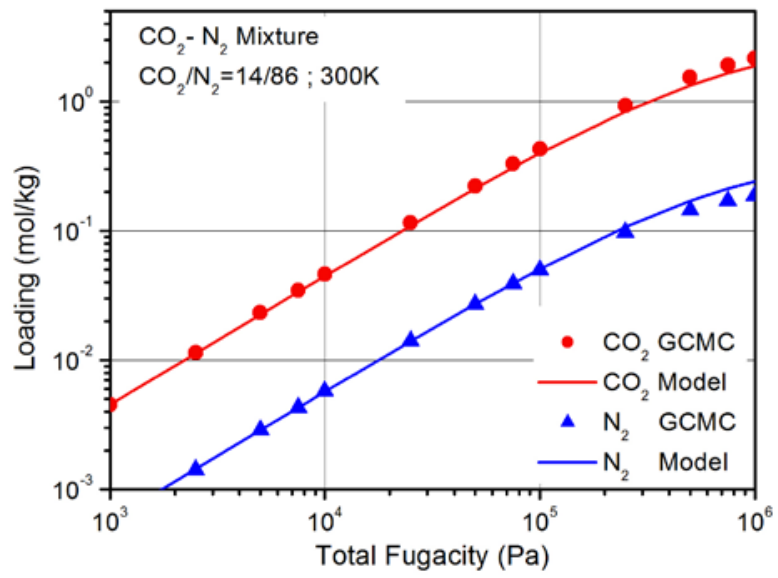

AFN

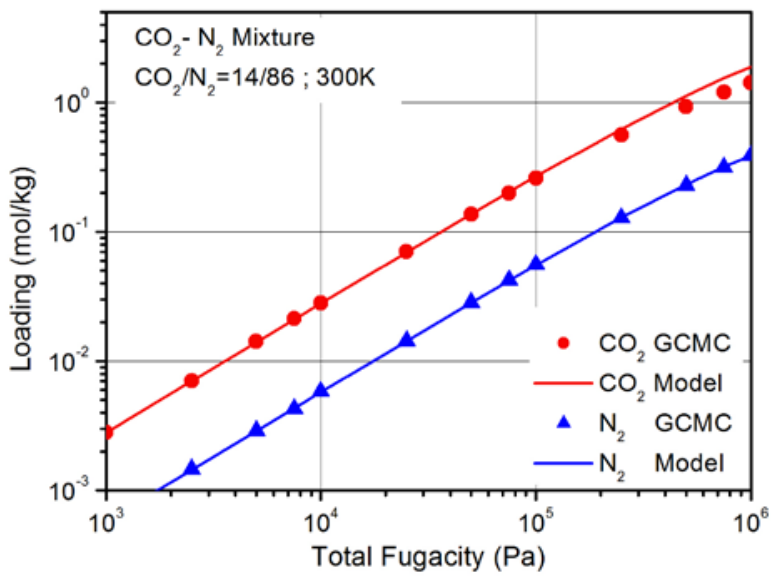

ACO 


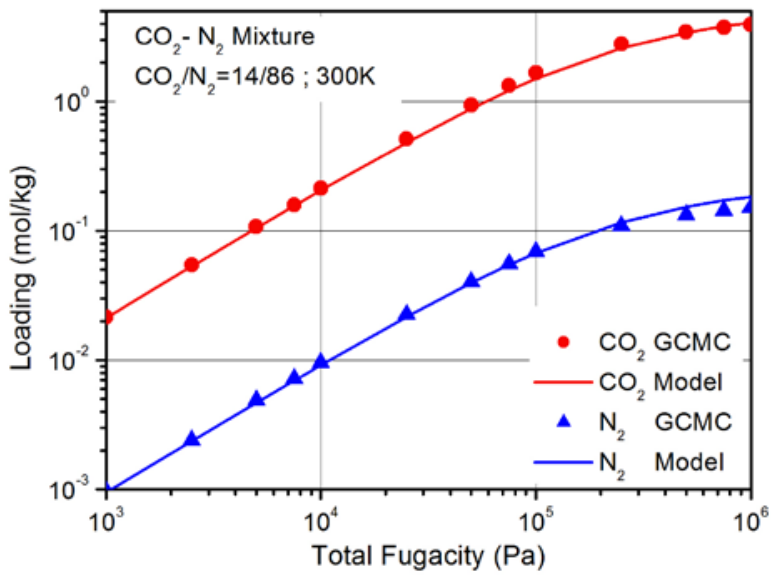

GIS

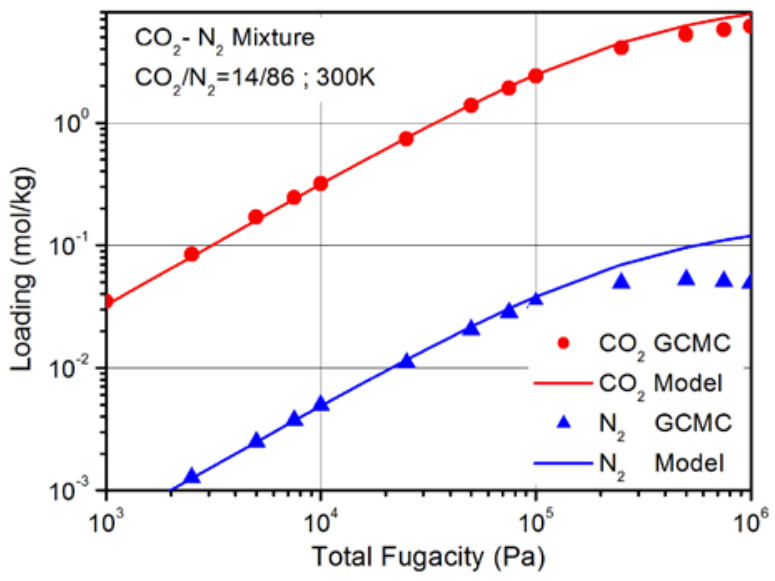

WEI 


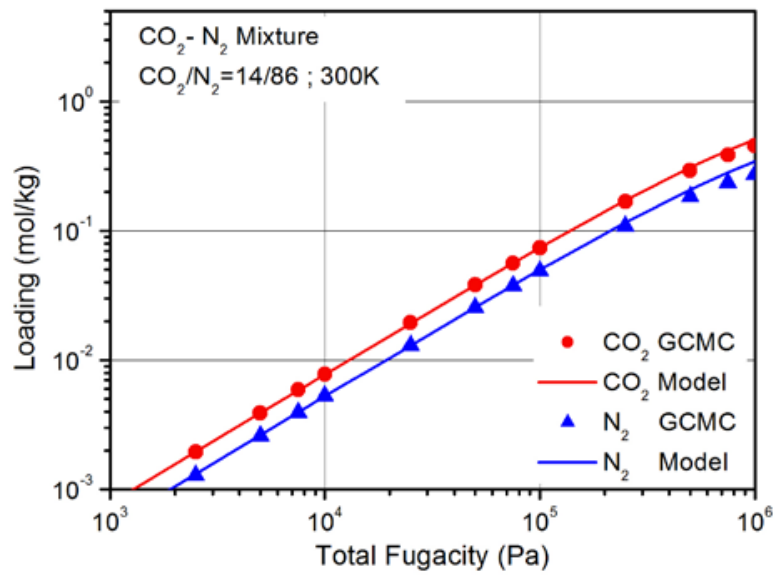

AEL

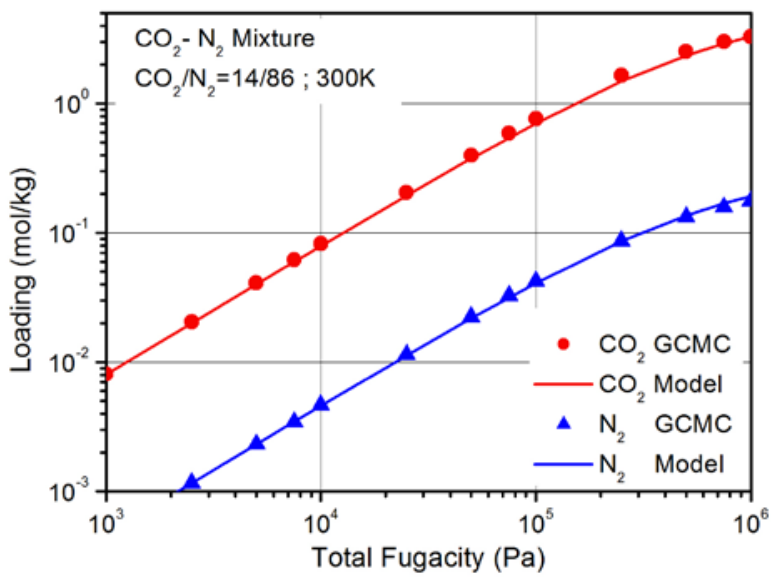

ABW 


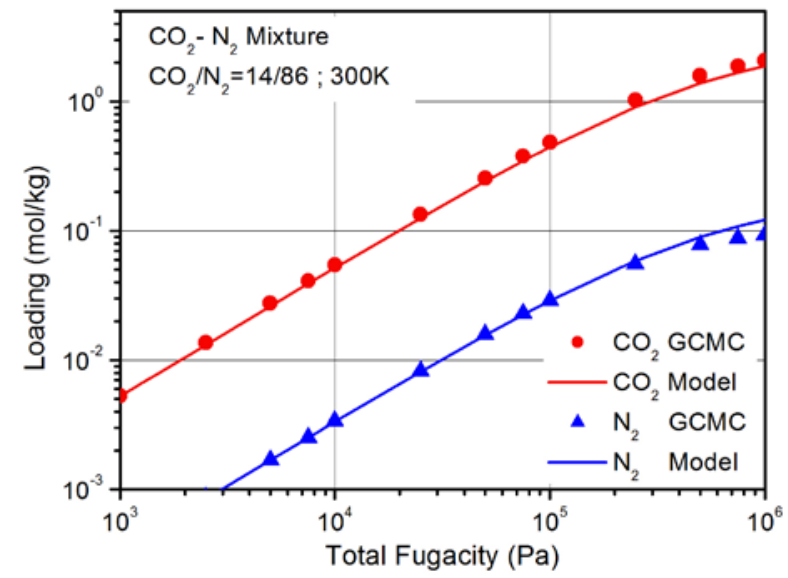

AEN

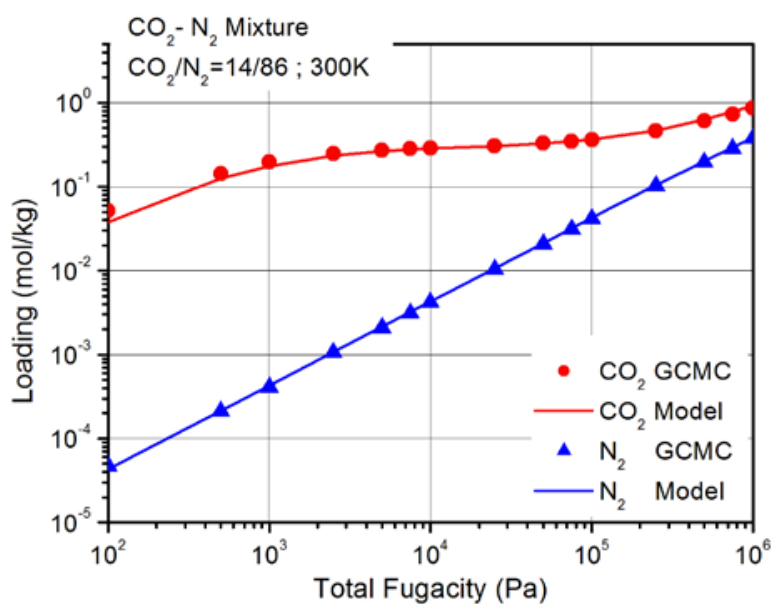

h8193735 


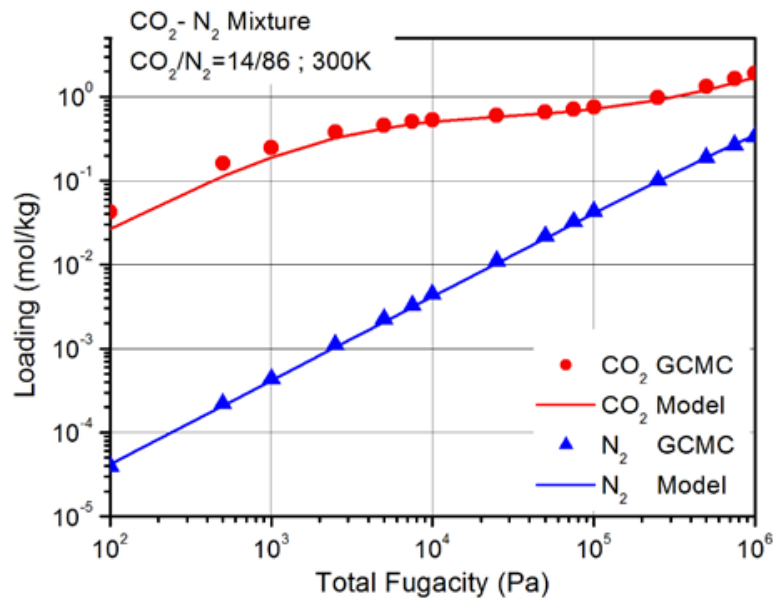

h8172492

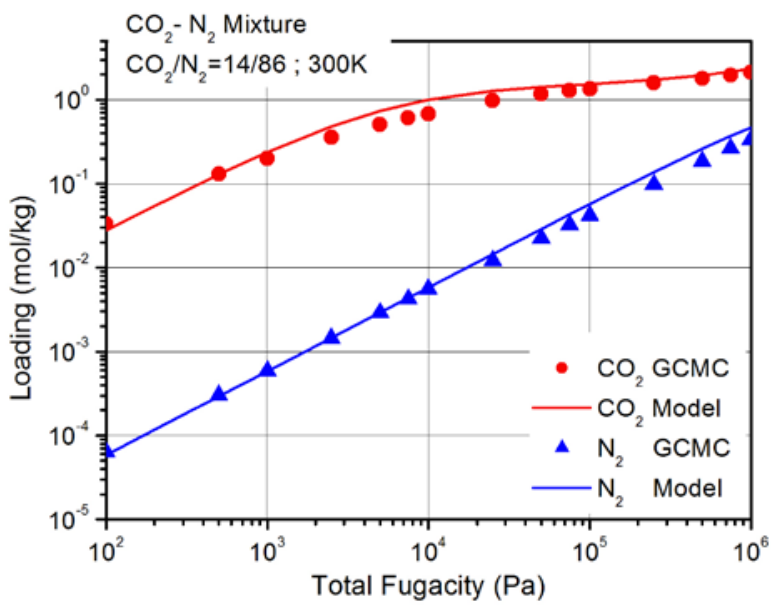

h8194737 


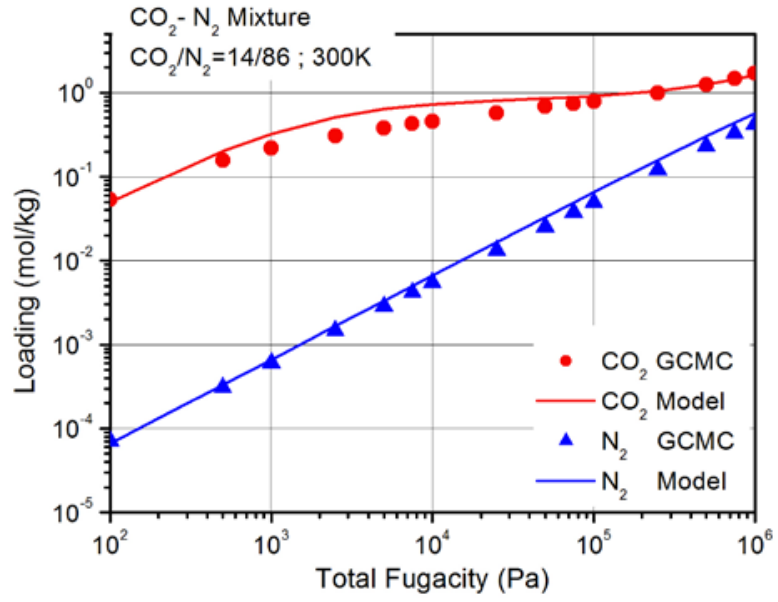

h819372

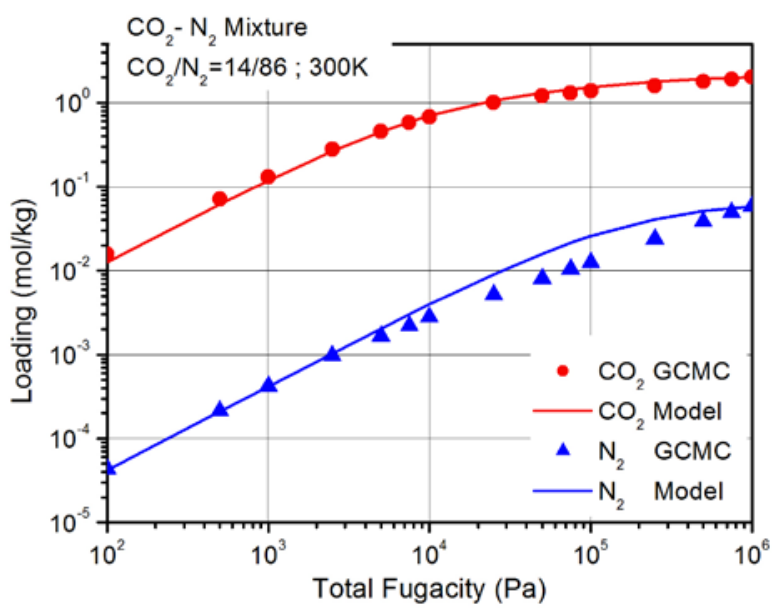

h8174128 


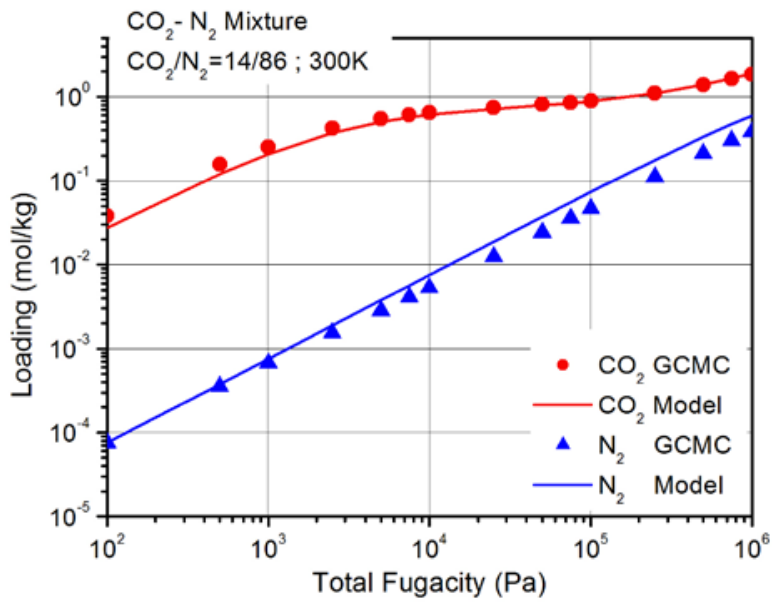

h8266159

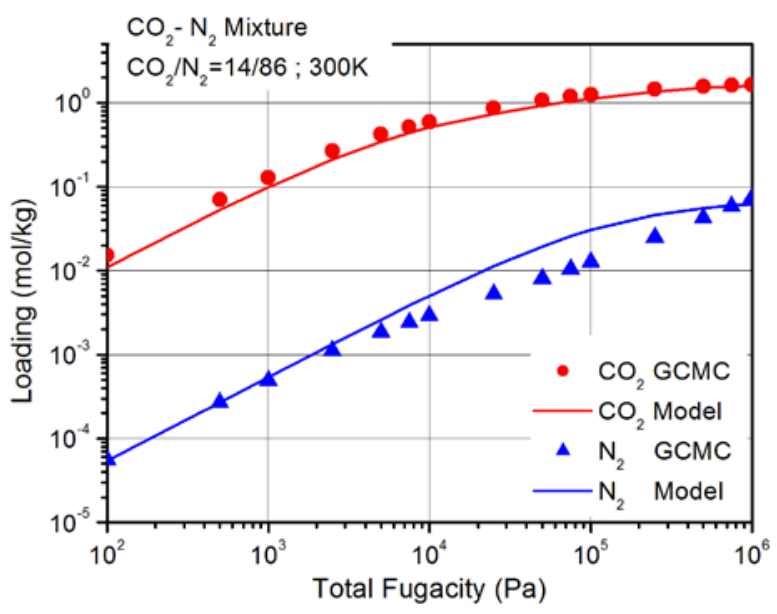

h8200029 


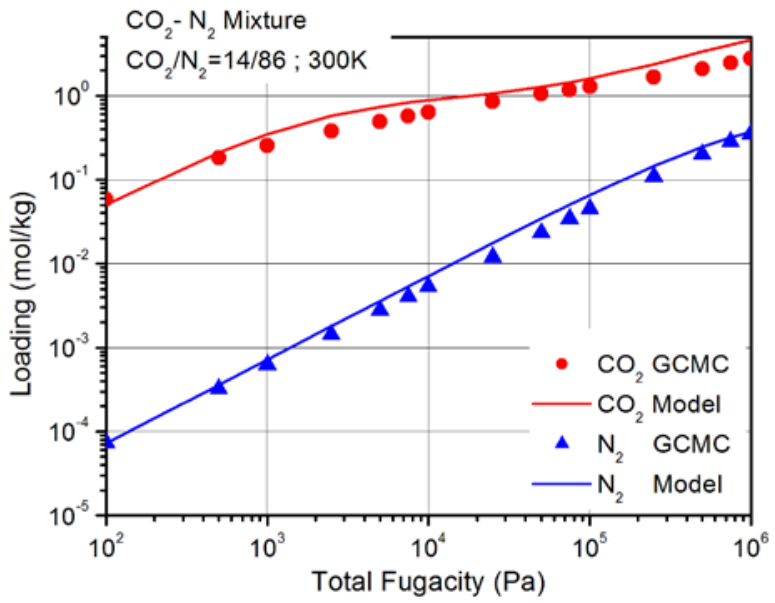

h8193722 